\title{
Author Index, Vol. 78, 1997
}

This index does not contain organizers and participants of the chromosome 1,10 and 12 mapping workshops which are published in this volume.

Cytogenetics and Cell Genetics

Achary, P.M. 46 Aguilera, M. 36 Almeida-Toledo, L.F. 236 Andersson, B. 21 Andréo,B. 202 Antignac,C. 240 Antonarakis, S.E. 213 Aoyama, T. 25 Arrigo, G. 145 Azzalin, CM. 112

Barclay, L. 120 Barnoski, B.L. 247 Bell,CJ. 247 Bertoni,L. 112 Bielec,P.E. 6 Bigner,S.H. 247 Blair,I.P. 140 Bockaert,J. 133 Bonaglia, M.C. 145 Bortoluzzi, S. 301 Bosma, A.A. 231 Boye,E. 240 Brookes, AJ. 21 Budarf,M.L. 247 Busbee, D.L. 6 Butcher, G.W. 50 Butler, L. 281

Call,K,M. 247 Capanna, E. 36 Carle, G.F. 65 Cass,I. 46 Ceccherini, I. 291 Chandley, A.C. 69 Charlieu, J.P. 202 Chen,H. 213 Chiu,I.-M. 48 Cho, K.-W. 135 Cinti,R. 31,291

Claeysen,S. 133 Cohen-Solal, L. 240 Coignet, L. 202 Colls, P. 27 Cortes, F. 197 Creaven, M. 31

Danieli, GA. 301 David, A. 240 Dawkins,J.L. 140 Deng,H.X. 63 Dobbie, L. 21 Dodds,K,G. 272 Douaire, M. 229 Dumuis, A. 133 Dunbar,D.R. 21

Egozcue, J. 27 Elder, F.F.B. 216 Eldridge, M.D.B. 74 Emanuel,B.S. 247 Ernst,,S. 120

Fagundes, V. 224 Faye,P. 133 Ferguson-Smith,

M.A. 260 Fernandes-Matioli,

F.M.C. 236 Feraandez-Piqueras,

J. 221 Fillon, V. 229 Flinter, F. 240 Flores, M.J. 197 Fredga,K. 253 Freier, S. 12 Fujiwara,T. 285,295 Fung,K,P. 105

Galloway, S.M. 272 Garagna, S. 36 Garcia-Barcelo, L.M.

105 Gaudray, P. 65 Gellin,J. 229 Gherzi,R. 145 Girardet,A. 202 Giulotto,E. 112 Goossens, F. 275 Gorodinsky, A. 289 Götz,H. 96 Gubler,M.C. 240

Haaf,T. 12 Haan,N.A.de 231 Haddad,L.A. 20 Harada,Y. 56 Harrison, W.R. 216 Hasegawa, A. 135 Hashimoto, T. 25 Hattori,T. 107 Hauwy,M. 147 Heidet,L. 240 Heitzmann, F. 31 Hendriks,D. 275 Henry, H.M. 272 Herranz, M. 221 Hill, A. 56 Horsley,D. 50 Hotta,Y. 69 Hou,J. 56 Hozier,J. 224 Hubert, R.S. 44 Hung, W.Y. 63 Hutchings, A. 50

Ikeuchi, T. 25 Inazawa,J. 103,209 Ishikawa, S. 58

Jenkins, Z.A. 272 Johnston, P.G. 74 Jost,E. 31 Juliano, L. 275 Juliano,M.A. 275

Kawabe, T. 61 Kawai,A. 285 Kehrer-Sawatzki, H.

96 Kemper,M.J. 240 Kimura, M. 107 Kinakin, B. 116 King, W. A. 124 Kingsley, K. 12

Kinlaw,W.B. 131 Klinger,H.P. 46 Ko,E. 120 Koga,M. 103 Kondo,N. 25 Kondoh,N. 103

Korenberg, J.R. 44 Krone, W. 96 Kyushiki,H. 285

Lamartine, J. 31 Lanfranchi, G. 301 Langlois,P. 229 Larget Piet, L. 240 Lee, C.Y. 105 Lefort, G. 202 Lemaire, S. 133 Lenoir, GM. 31 Leprini, A. 145 Lin, A.H. 275 Liu, Y. 48 Luk, S.C.W. 105 
Ma, A. 50

Maarel, S. van der 12 McQuade,L.R. 74 Macville,M. 281 Mäkinen, A. 231 Maloney, M. 131

Martin, R.H. 116,

120 Martínez-Pasarell, O.

27 Marziliano, N. 36 Mascheretti, S. 36 Masuda,A. 209 Matsuda,Y. 107 Matsumiya, K. 103

Mayer, J. 1

Meese, E. 1 Meléndez,B. 221 Mellink, C.H.M. 231 Merril,C.R. 281 Metcalfe, C.J. 74

Milbrandt,J. 289 Miyajima, A. 209 Mohandas, T.K. 131,

247 Moncur,J.T. 131 Montgomery, G.W.

272 Mucciolo, E. 112 Mueller-Lantzsch, N. 1 Müller, S. 260 Mullokandov, M. 46 Muto,T.

295

Nakamura, Y. 58,

285,295 Nakashima, S. 107 Namiki,M. 103 Narain,Y. 253 Nicholson, G.A. 140 Nishimune, Y. 103 Nishina,Y. 103 Nogués, C. 27 Nojima,H. 103 Nozaki,M. 103

O’Brien, P.C.M. 260 Ogawa, M. 58 Okamoto, T. 61 Okano,Y. 107 Okuyama, A. 103

Omori,Y. 285 Ono,T. 61 Orii,K.O. 25 Orii,T. 25 Ortiz, T. 197 Osada,H. 209 Otte,A.P. 50

Parente, F. 65 Park, J.P. 131,247 Pellestor,F. 202 Pena,S.D.J. 20 Perez de Castro, I.

221 Pérez-Zapata, A. 36 Piñero,J. 197 Pontarotti, P. 137 Popescu,N.C. 289 Puliti,A. 291

Rademaker, A. 116,

120 Radomski, N. 31 Rambau,R.V. 216 Rampoldi,L. 301 Redi,C.A. 36 Rhodes, C.H. 247

Riggs,P.K. 6 Riviere, M. 137 Robinson, T.J. 216 Röck,H. 96 Romeo, G. 31,291 Ropers, H.H. 12 Rumpler, Y. 147

Saito, T. 209 Saito-Ohara, F. 25 Santos, J. 221 Sasaki, S. 295 Satoh,H. 135 Scalzi-Martin, J.M.

224 Scharpé,S. 275 Schatteman, K. 275 Sebben,M. 133 Seri,M. 31,291 Shibasaki,Y. 21

Siddque,T. 63 Siegel,A. 96 Sims,K. 224 Singh, P.B. 50 Sonta, S. 61 Speed, R.M. 69

Sumi,N. 107 Summers, N. 120 Suzuki, M. 285 Sylla,B.S. 31 Szpirer,C. 137 Szpirer, J. 137

Takahashi, E. 56,

285,295 Takahashi,T. 58,209 Takase,H. 69 Takeda,S. 285 Takei,Y. 295 Taketo,M. 103

Tanaka,H. 103 Taviaux, S. 133 Tazi,R. 137 Templado, C. 27 Thorner, P. 240 Tiso,N. 301

Toledo-Filho, S.A.

236 Tsuchida, J. 103 Tsui, S.K.W. 105 Tsujimoto, H. 135

Tumennasan, Kh. 69 Turc-Carel, C. 65 Tuya, Ts. 69

Uchida,K. 103,209 Umeda,Y. 107

Valle,G. 301 Vanhoof,G. 275 Verrinder Gibbins,

A.M. 124 Vezuli,A. 147 Vidal,F. 27 Vignal,A. 229

Wagner, M.J. 56 Wang, G 50 Warier, S. 147 Watari,T. 135 Waye,M.M.Y. 105 Wells, D.E.

56 Wienberg,J. 260 Wilkes,C. 281 Wirth,J. 12 Womack, J.E. 6

Xia,J.H. 63 Xu,L. 63

Yanagisawa, K. 209 Yang,J.-P. 61 Yang,Y. 63 Yonenaga-Yassuda,

Y. 224 Yoshimura, A. 209 Yoshioka,T. 107 Youn,H.-Y. 135

Zahorchak, R.J. 281 Zardi,L. 145 Zhang, X. 240 Zhou,J. 240 Zijlstra,C. 231 Zimbello,R. 301

Zimmer, R. 124 Zimonjic, D.B. 289 Zuccotti, M. 36 Zuffardi,O. 145 Zullo,S.J. 281

KÄIUiER

E-Mail karger@karger.ch Fax + 4161306 1234 
(C) 1997 S. Karger AG, Basel 